\title{
A Holistic Supplementation Regimen for Tongue-Tied Babies With Slow Weight Gain and Failure to Thrive
}

Share this:

\author{
Asti Praborini, MD, IBCLC, RLC ${ }^{a}$ \\ Anjar Setiani, $\mathrm{MD}^{\mathrm{b}}$ \\ Agusnawati Munandar, MD, IBCLC, CIMI, RLC \\ Ratih Ayu Wulandari, MD, IBCLC, RLC ${ }^{\mathrm{d}}$
}

\begin{abstract}
Breastfeeding is the best way to feed infants, but optimal milk transfer and weight gain depend on good latching. Tongue- and lip-tie can prevent successful latching and prevent adequate nutrition. Tongue- and lip-tied babies can either have slow weight gain (SWG) or failure to thrive (FTT). We examine the effect of a holistic supplementation regimen on tongue-tied babies with SWG and FTT. This was a descriptive, cross-sectional study of 55 tongue- and lip-tied babies with SWG and FTT at KMC Hospital, Jakarta, Indonesia. All babies underwent frenotomy and received supplementation with formula (64\%) or pasteurized donor breast milk, using either a modified lactation aid $(78 \%)$ or the Medela Supplemental Nursing System (22\%). All mothers received domperidone and acupuncture to improve milk supply. A majority of babies had type 3 tongue-tie (46\%) and class 3 upper lip-tie. Twenty-five subjects (45\%) had SWG, and 30 subjects (55\%) had FTT. All mothers had low milk supply. At-the-breast supplementation improved the nutritional status of $44 / 55$ subjects $(80 \%, \mathrm{p}<.001)$, whereas 11 subjects received early complementary feeding at 4 months of age. By the end of the study, all subjects were solely breastfed without at-the-breast supplementation. The holistic management of tongue-and lip-tied babies with SWG or FTT consisting of frenotomy, at-the-breast supplementation, domperidone, and acupuncture improved infant nutritional status and the mother's milk supply. Babies could breastfeed without supplementation after treatment and gained weight.
\end{abstract}

Keywords: slow weight gain; failure to thrive; breastfeeding; tongue-tie; lip-tie; at-the-breast supplementation

A child's nutrition in the first 2 years of life is particularly important, with optimal nutrition during this time associated with improved short- and long-term morbidity and mortality, reduced risk of chronic disease, and overall better development. Undernutrition is estimated to be associated with 2.7 million child deaths annually, accounting for $45 \%$ of all child deaths. Therefore, infant and young child feeding is a key area to improve child survival and promote healthy growth and development (World Health Organization, 2016).

Breastfeeding provides optimal nutrition while initiating bonding and providing warmth and security. Breastfeeding has numerous health benefits over formula, and there is abundant evidence that breastfeeding reduces the risk of a wide range of infectious diseases including bacterial meningitis, bacteremia, diarrhea, respiratory tract infections, otitis media, urinary tract infections,

a. astipraborini@yahoo.com

b. anjarsetianispa@gmail.com

c. wati_arkan@yahoo.com

d.dr.ratih@menjadiibu.com and late-onset sepsis in preterm infants. Breastfeeding also decreases the risk of developing childhood malignancy and allergic and metabolic diseases, such as asthma, diabetes mellitus, and hypercholesterolemia (Gartner et al., 2005; Horta \& Victora, 2013).

Since breastfeeding represents a collaboration between mother and infant in their dyad, support for optimal breastfeeding must be equal for both mother and baby and might include family, physicians, and allied hospital staff caring for both mother and baby. Mothers should be taught how to position and latch her baby from the start of breastfeeding, and any problems with milk transfer need to be examined (Neifert, 2004).

Successful breastfeeding depends on a correct latch. For newborns, the chin should rest on the breast below the nipple, the lips should be everted, and at least the lower lips should flange outward (Kotlow, 2011). Anatomical factors might prevent a baby's mouth from allowing optimal milk transfer; for example, an overly turnedout upper lip is a sign of a shallow latch and can favor 
excessive use of the lips to press milk out of the breast, and a tight superior labial frenulum can make it more difficult to maintain latching (Genna, 2013). Tongue-tie is a well-recognized cause of breastfeeding difficulties that can prevent the baby from latching well, cause nipple pain and trauma, compromise feeding, inhibit weight gain, and, in some cases, reduce maternal milk supply (Garbin et al., 2013).

A baby with optimal milk transfer is expected to gain weight in line with the World Health Organization (WHO) exclusively breastfed growth curves (Powers, 2004). However, a breastfeeding baby with ineffective latching may have slow weight gain (SWG) or fail to thrive. SWG is defined as (a) an infant aged less than 2 weeks who is more than $10 \%$ less than birth weight or (b) who is aged 2 weeks to 3 months but whose weight gain is less than $20 \mathrm{~g} /$ day (Walker, 2011a). Failure to thrive (FTT) is defined as an infant with body weight under the 3rd percentile or Z-score $<-2$, and FTT occurs when an infant continues to lose weight after 10 days and does not return to birth weight by the age of 3 weeks or remains below the 10th percentile by the end of the first month (Powers, 2004). Undernutrition during the first year may have long-term effects on the developing brain and impair intellectual development so must be addressed (Alamy \& Bengelloun, 2012; Ivanovic et al., 2000; Scrimshaw, 1998).

Frenotomy, which describes the cutting of the lingual frenulum, has been shown to effectively resolve breastfeeding difficulties in infants with tongue-tie in clinical studies (Garbin et al., 2013; Kumar \& Kalke, 2012). Early frenotomy in an infant during lactogenesis II prior to day 8 appears to have a larger impact on weight gain and lactation performance than late frenotomy after Day 8 , which while improving latching, only results in small weight increases due to reduced maternal milk supply (Praborini, Purnamasari, Munandar, \& Wulandari, 2015). Therefore, following frenotomy, we suggest a combinatorial treatment in a baby with nutritional problems and a mother with low milk supply consisting of supplementation with lactation aid, domperidone, and acupuncture.

Supplementation is defined as the practice of giving the breastfed infant additional nutrition other than that obtained directly from the breast. This may include expressed or banked breast milk and/or breast milk substitutes/formula (Riordan, 2005; The Academy of Breastfeeding Medicine Protocol Committee, 2009). Any foods given prior to 6 months (the recommended exclusive breastfeeding duration) are thus defined as supplementary (The Academy of Breastfeeding Medicine Protocol Committee, 2009). The WHO only recommends giving a child complementary foods between 4 and 6 months if he or she (a) is not adequately gaining weight despite appropriate breastfeeding or (b) receives frequent breastfeeds but appears hungry soon after (WHO, 2000). In our regimen, we also add domperidone and acupuncture to increase the mother's milk supply with the intention that after a period of time, the increase in mother's milk supply will allow supplementation to be decreased, and then stopped.

Here, we examined the effect of supplementation on babies with SWG and FTT due to tongue-tie. We demonstrate that the multi-modal management of tongue-tied babies with SWG or FTT with frenotomy, supplementation, domperidone, and acupuncture improves the child's nutritional status and also the mother's milk supply. After treatment, babies breastfed without supplementation and gained weight with breastfeeding alone.

\section{Method}

\section{Study Setting and Lactation Team}

The study setting was a mother and children's hospital in Jakarta, Indonesia. The hospital operates a lactation clinic 12 hours daily, run by physicians who also act as lactation counselors/consultants in three shifts per day. All lactation doctors have undergone a 40-hour breastfeeding counseling course and courses on lactation management, complementary feeding for breastfed babies, and kangaroo mother care.

In our unit, the lactation doctor consults patients in a lactation clinic and conducts postnatal rounds to see mothers and infants hospitalized because of nipple confusion, mastitis, abscess, or other lactation problems, such as nursing strike or adoptive nursing. The lactation team consists of physicians, dentists, and pediatricians and is led by a pediatrician registered with the IBCLC. Patients with breastfeeding problems can present to either lactation clinic or pediatric clinic. Cases are reported to the team leader on a daily basis for case direction to progress cases. The lactation team also meets regularly for journal reading and other educational activities.

\section{Study Participants}

All files of eligible individuals attending pediatric or lactation clinic between March 2013 and September 2014 were reviewed. The inclusion criteria were tonguetied babies with SWG or FTT. Exclusion criteria were babies with nipple confusion, congenital heart disease, other congenital anomalies, or other problems that 


\section{Figure 1. Inclusion and exclusion criteria.}

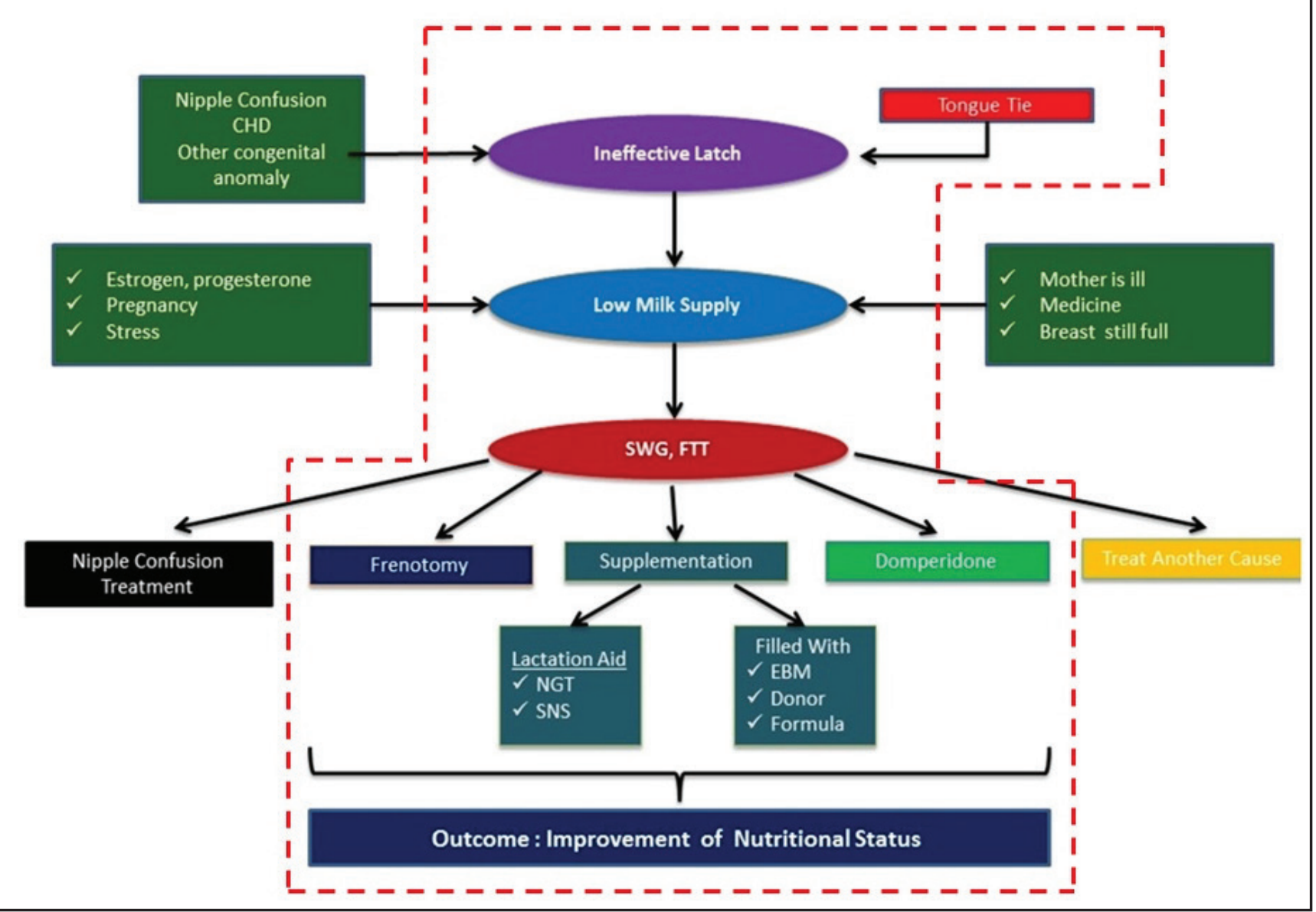

Note. The scope of the study is indicated within the dashed line.

interfere with breastfeeding (Figure 1). Sixty-nine cases were identified, of which 14 were excluded due to loss to follow-up, leaving 55 cases available for study. The subject characteristics are shown in Table 1 .

Mothers and infants presented with various complaints. Mother complaints included pain during breastfeeding, feeling of an empty breast, feeling that her baby was not satisfied during breastfeeding, and feeling exhausted due to continuous breastfeeding throughout the day and night. Infant problems included latching only to the nipple, having long breastfeeding periods without satiety, excessive weight loss, or not achieving the recommended weight gain.

A thorough history and examination of both mother and baby were performed in all cases. For the mother, the breasts and nipples were inspected, palpated, and her milk supply was also checked. The baby's general appearance and mouth were examined. Babies with SWG will look alert and healthy, have good muscle tone and skin turgor, and have at least six wet diapers per day with frequent stools. Baby with FTT will look apathetic or crying, have poor tone and skin turgor, few wet diapers, concentrated urine, and infrequent stools (Mannel \& Martens, 2013). We also evaluated the breastfeeding dyad and baby's mouth. Tongue-tie and lip-tie significantly interfered with the breastfeeding dyad.

Tongue-tie was classified according to the Coryllos classification: type 1 , the tip of the tongue is in front of the gums; type 2, 2-4 $\mathrm{mm}$ behind the tip of the tongue, attached to or slightly behind the gums; type 3 , attached to the middle of the tongue to the floor of the mouth; and type 4 , at the base of the tongue, thick, shiny, and not very elastic (Coryllos, Watson Genna, \& Salloum, 2004).

Lip-tie was grouped according to the Kotlow classification: class I, requires no treatment; class II, attaching at the free junction and attached gingival tissue; class III, attaching just in front of the anterior papilla; and class 


\section{Table 1. The Characteristics of the Study}

\section{Population}

\begin{tabular}{|l|l|}
\hline & $n(\%)$ \\
\hline Sex & \\
\hline Male & $26(47)$ \\
\hline Nutritional status & $29(53)$ \\
\hline Slow weight gain & \\
\hline Failure to thrive & $25(45)$ \\
\hline Type of tongue-tie & $30(55)$ \\
\hline Type 1 & \\
\hline Type 2 & $6(11)$ \\
Type 3 & $10(19)$ \\
Type 4 & $25(46)$ \\
\hline Class of lip-tie & $14(24)$ \\
\hline Class 1 & $12(22)$ \\
\hline Class 2 & 0 \\
Class 3 & 0 \\
\hline Class 4 & $46(83)$ \\
\hline Lactation aid & $9(17)$ \\
\hline Modification of nasogastric tube and \\
Syringe
\end{tabular}

IV, attaching at the anterior papilla and extending into the hard palate (Kotlow, 2011). Babies' weights were plotted using the WHO Anthro growth and development book.

We divided our subjects into two groups, SWG and FTT, both receiving the same holistic regimen (frenotomy, supplementation, domperidone, and acupuncture). We evaluated relationships between weight gain and age and weight gain and duration of treatment for each group by multiple regression. All data were analyzed with SPSS version 19.0 (IBM Statistics, Chicago, IL).

\section{Procedure}

Parents attending pediatric or lactation clinics received a full explanation of the tongue-tie and lip-tie causing SWG or FTT. The treatment options including frenotomy, tongue and lip exercises, tummy time, supplementation, domperidone treatment, and acupuncture were explained to parents. Participant mothers and babies were advised to attend weekly follow-up. Mothers provided written informed consent prior to the procedure.

Frenotomy was performed either in the pediatric or lactation clinic. Babies undergoing frenotomy in the pediatric clinic were subsequently referred to lactation clinic for tongue and lip exercises, tummy time, and at-the-breast supplementation titration.

For frenotomy, the baby was swaddled to immobilize the arms and legs and laid supine on the examination table. An assistant held the head still while the operator lifted the tongue with a finger to locate the problematic frenulum. The frenulum was then snipped with blunt-ended sterile scissors, and sterile gauze was used to attain hemostasis. The tongue-tie was deemed completely released if a neat diamond shape was visible with no palpable tissue remaining to restrict tongue movement. We favored not using anesthesia to perform the procedure because it can subsequently delay breastfeeding.

Immediately after frenotomy, the mother was asked to breastfeed for re-evaluation of latch. The patient was then referred to lactation clinic for titration of supplementation.

The lactation doctor gave the mother different options for supplementation, either a modified lactation aid (with a $50 \mathrm{cc}$ syringe plus nasogastric tube $5 \mathrm{Fr} 40 \mathrm{~cm}$; Figure 2A) or the Medela Supplemental Nursing System (Medela, Baar, Switzerland; Figure 2B). These feeding tube devices have the advantage of supplying appropriate supplement while simultaneously stimulating the breast to produce more milk and reinforcing the infant's feeding at the breast (The Academy of Breastfeeding Medicine Protocol Committee, 2009). The mother was taught how to prepare and clean their chosen feeding tube device.

The lactation doctor also provided the mother with different options for supplementation: either pasteurized donor breast milk or formula. If the mother had 


\section{Figure 2. (A) Supplementation using the modified lactation aid. (B) Supplementation using the supplemental nursing system.}

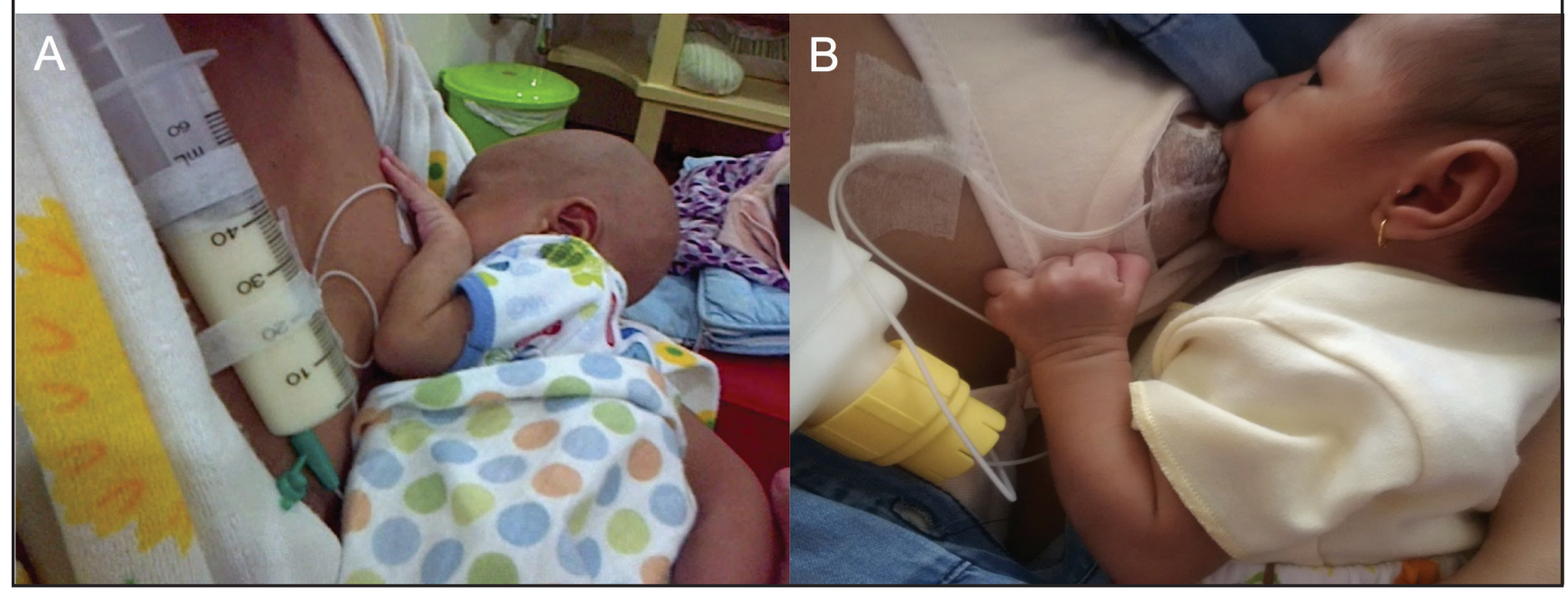

pumped or expressed her milk before supplementation and had refrigerated storage, she could use it as supplementation. In the absence of storage, mothers were discouraged from pumping or expressing their breast milk during the supplementation period.

Breastfeeding with a lactation aid device can be regarded as drinking from two cups: the breast and the feeding tube device. Once the breast becomes fuller due to the effects of domperidone and acupuncture, the other "cup," that is, the amount of supplementation in the feeding tube device can be decreased. We advised the mothers to not pump during supplementation since this would empty the breast and necessitate more compensatory supplementation from the feeding tube device to fill the baby. In the authors' experience, pumping interferes with a baby's weight gain and supplementation cannot be decreased, but instead needs to be increased and cannot be stopped despite of the literature suggesting that pumping increases milk production (Riordan, 2005; Wambach, 2014). We also found that most mothers consider this method exhausting, making them more likely to give up. The aim of this program was to decrease and finally stop supplementation over time.

To assess the need for supplementation, the lactation doctor titrated the amount of milk required using the feeding-tube device. Prior to breastfeeding, the lactation doctor placed a known amount of milk inside the feeding tube device and taped it at the breast before letting the baby breastfeed. After the baby had finished and was fully sated, the amount of supplementation needed was calculated. For example, if $90 \mathrm{cc}$ of milk was inside the feeding tube device at the start of feeding and $30 \mathrm{cc}$ milk remained inside the feeding tube device after feeding, an additional $60 \mathrm{cc}$ of milk was needed per breastfeed. On this basis, we advised the mother to supplement with the feeding tube device six times per day (e.g., $6 \times 60 \mathrm{cc}$ ) from early morning until 9:00 pm at the latest, with the mother only breastfeeding without the feeding tube device overnight to prevent exhaustion.

In addition, the lactation doctor taught the mother how to perform tongue and lip exercises to prevent reattachment and promote tummy time for better breastfeeding. Tummy time has been shown to be beneficial for breastfeeding (Wen, Baur, Simpson, Rissel, \& Flood, 2011).

Domperidone and acupuncture were also part of the regimen. Domperidone is a dopamine D2-receptor antagonist that rapidly increases milk volume within 48 hours. Domperidone increases daily milk production in lactating women with insufficient milk production by stimulating prolactin. Small amounts of domperidone are excreted into breast milk, but nursing infants experience no adverse events. Over the course of treatment, mothers must slowly taper the dose to avoid withdrawal effects (Albright, 2004; Briggs, Freeman, \& Yaffe, 2011; Jones \& Breward, 2011; van Paassen et al., 2016). Ten 20-mg domperidone three times daily was prescribed depending on the case.

Mothers were referred to acupuncture specialists and received serial acupuncture treatment. Acupuncture is an ancient and effective treatment used in China for insufficient milk production. Acupuncture has a strong influence on systemic hormone levels, and it is thought that its positive effects on lactation may be due to its increasing oxytocin and prolactin levels (Walker, 2011b; Yelland, 2005). 


\section{Table 2. Details of the Supplementation Given}

\begin{tabular}{|l|l|l|l|}
\hline Data & Median & Minimum & Maximum \\
\hline Age upon first consultation (days) & 32 & 5 & 119 \\
\hline Duration of supplementation (days) & 41 & 6 & 183 \\
\hline Weight gain (grams/day) & 32 & 9 & 183 \\
\hline
\end{tabular}

Domperidone, acupuncture, and optimal latching after frenotomy will stimulate the breast to produce milk. Mothers were scheduled to receive follow-up every week to evaluate weight gain by plotting babies' weights in WHO Anthro growth and development books to decide the need for and amount of supplementation. The decision to continue, increase, or decrease supplementation was based on weight gain. Weight gain was calculated according to weight on review minus body weight a week before and divided by days. If we determined that a baby's weight gain per day was already sufficient according to their Anthro growth, we decreased the supplementation $10 \mathrm{cc}$ per feed (e.g., $6 \times 60 \mathrm{cc}$ to $6 \times 50 \mathrm{cc}$ ) and advised the mother to return a week later for further review. The duration of supplementation is shown in Table 2.

Each treatment varied according to the individual mother-baby dyad. Treatment was also dependent on the mother's background. For example, working mothers compared to stay-at-home mothers. Maternity leave in Indonesia is only 3 months and must be taken 1.5 months before delivery and 1.5 months after delivery. If a baby has SWG or FTT, we can write to the employer to recommend additional leave for 30 to 60 days. In cases where it was impossible to obtain additional leave, the mother could still breastfeed with the feeding tube device 2-3 times at home, with the baby receiving supplementation via cup feeding to avoid nipple confusion while at work. At 4 months, we suggested early complementary feeding, rather than supplementation with formula, to encourage good weight gain while continuing breastfeeding.

\section{Results}

Fifty-five cases were available for study. Most subjects $(51 / 55,93 \%)$ were born outside the hospital, and four were born in our hospital. Forty-seven percent were male, and 53\% were female. Of 55 babies, 25 (45\%) had SWG, and 30 babies (55\%) had FTT. The median baby age at consultation was 32 days (range 5-119 days).

As shown in Table 1, the distribution of tongue-tie was type $3(46 \%)$, type 4 (24\%), type $2(19 \%)$, and type $1(11 \%)$.
For upper lip-tie, babies were class 3 in $83 \%$ of cases and class 4 in $17 \%$ of cases. All parents agreed to frenotomy, tongue, and lip exercises and treatment with supplementation. There were no bleeding, infection, or reattachment complications.

All subjects received supplementation to increase their body weight. Forty-three subjects $(78 \%)$ used the modified lactation aid with syringe and nasogastric tube due to its cheaper cost, and 12 subjects (22\%) used the Medela Supplemental Nursing System.

Thirty-five subjects chose formula because it was easier to obtain compared to pasteurized donor milk (in 14 subjects). Some subjects also considered milk kinship since there is no human milk bank in Indonesia. Five subjects mixed both formula and pasteurized donor milk, and one subject used mother's expressed breast milk due to excess breast milk. However, during supplementation, she did not continue pumping.

All mothers received domperidone and acupuncture. The median duration of supplementation was 41 days (range 6-183 days). With therapy, weight increased by a median of $32 \mathrm{~g} /$ day (range 9-183 g/day). There were significant improvements in nutritional status from before to after supplementation $(p<.001)$.

All SWG babies (30 subjects) had adequate weight gain and their nutritional status improved as well as growth.

We next analyzed the relationship between weight gain before and after therapy and age and duration of treatment in the SWG group using a multiple regression model. Age and duration of treatment together explained $54 \%$ of the weight gain $(p<.0001)$, with age negatively associated with weight gain $(p=.04)$ and duration of treatment positively associated with weight gain $(p=.001)$. For the FTT group, age and duration of treatment explained $86 \%$ of the weight gain $(p<.0001)$, with age negatively but not significantly associated with weight gain $(p=.71)$ but duration of treatment positively associated with weight gain $(p<.0001)$. 
Of 25 babies with FTT, 19 (63\%) gained weight, but 11 babies (37\%) did not show adequate weight gain. These 11 subjects received early complimentary feeding at 4 or 5 months of age. Complementary foods were those advised by the WHO and UNICEF and included a variety of foods at each meal. For example, 1 star $^{*}$ animal-based foods (meat, eggs, and dairy products); 2 stars ${ }^{* *}$ staples (grains, roots, and tubers); 3 stars $^{* * *}$ legumes and seeds; 4 stars $^{* * *}$ vitamin A-rich fruits and vegetables and other fruits and vegetables, with additional water and fat from oil, butter, or coconut milk (UNICEF, 2016; WHO, 2016). Supplementation was stopped after complementary feeding started and baby was only breastfeeding. These babies went on to gain weight, and their nutritional status improved. By the end of the study, all subjects were growing well and continuing breastfeeding without supplementation.

\section{Discussion}

Babies with SWG or FTT are usually supplemented with formula and bottle, or nasogastric tube inserted into the stomach, to improve their nutritional status. Formula dosages are increased based on the baby's age and weight. However, this method is likely to cause nipple confusion, reduce the mother's milk production and self-esteem, and result in early weaning from the breast.

Here we used an at-the-breast supplementation program to optimize breastfeeding. Feeding tube devices have

\section{Figure 3. (A) An SWG baby who became well-nourished after frenotomy and}

supplementation. (B) An FTT baby who became well-nourished after frenotomy and supplementation without early complimentary feeding. (C) An FTT baby who became wellnourished after frenotomy, supplementation, and early complimentary feeding.
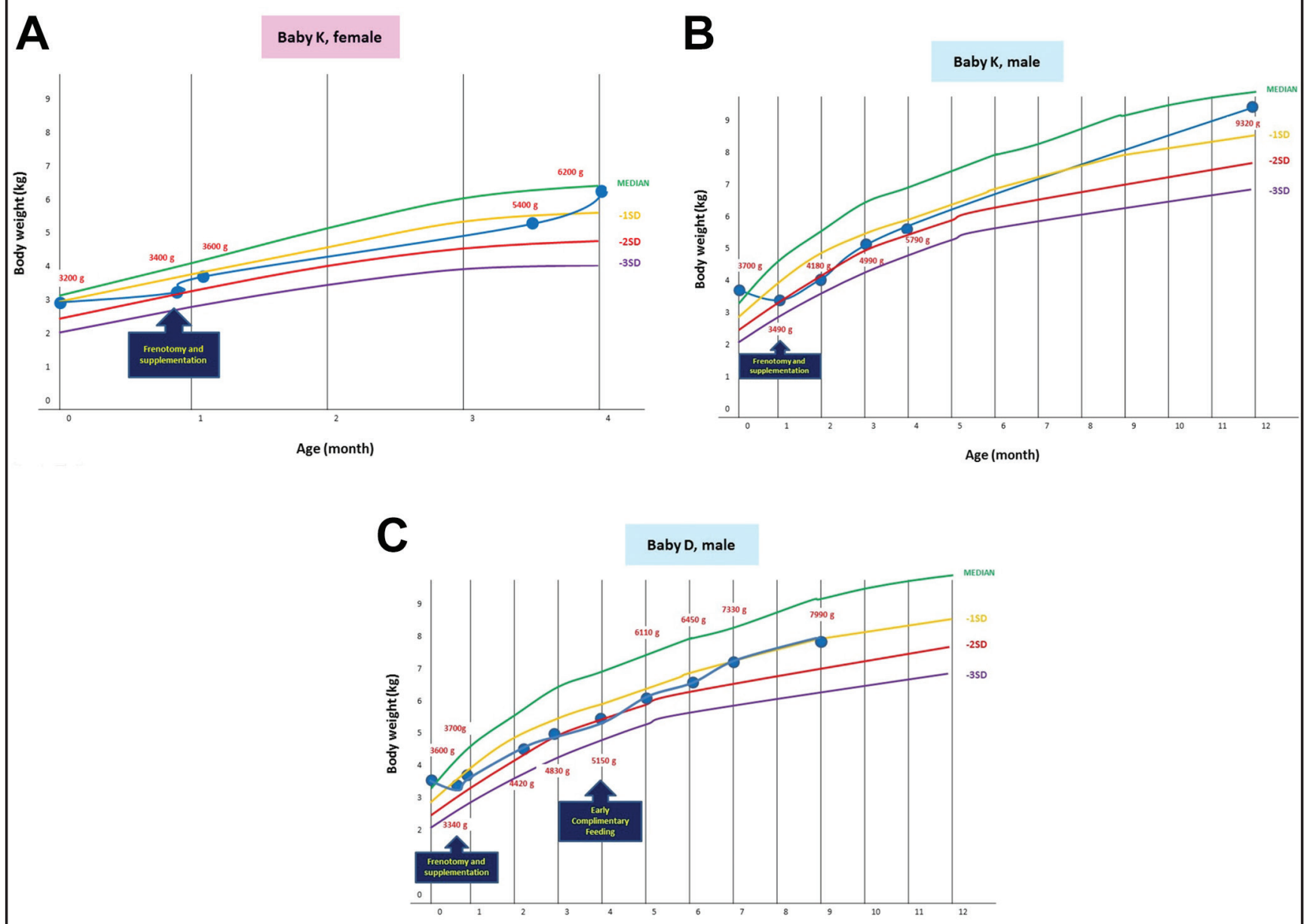

Age (month) 
the advantage of supplying an appropriate supplement while simultaneously stimulating the breast to produce more milk and reinforcing the infant's feeding at the breast. Optimal latching after frenotomy, tongue and lip exercises to prevent re-attachment, tummy time for better breastfeeding, domperidone, and acupuncture represented a holistic approach to increase mother's milk production. Over time, as the milk supply increased, pasteurized donor or formula milk dosages could be decreased and finally stopped.

Forty-six percent of subjects had type 3 tongue-ties, and $25 \%$ had type 4 ties, the former apparently causing greater problems with milk transfer and consequent SWG and FTT. This is consistent with data showing that the degree of posterior tongue depression is extremely important in dictating the quantity of milk transferred per suck. The grooved tongue collects the milk into a bolus, which in young infants is delivered to the pharynx on every swallow (Genna, 2013). Infants with type 3 tongue-ties find this role difficult.

Eighty-three percent of subjects in our study had class 3 lip-tie, and 17\% had class 4 lip-tie. Classes 3 and 4 lip-tie may contribute to breastfeeding problems and require revision (Kotlow, 2011). Similar to our population, Pransky, Lago, and Hong (2015) also reported frequent anterior and posterior tongue-ties, upper lip-ties, or their combinations, with upper lip-tie release improving breastfeeding in all cases.

Our analysis showed that, for the SWG group, the younger the baby started treatment, the higher the weight gain. The age the treatment started did not relate to weight gain for the FTT group, instead the baby's weight closely related to the length of treatment. This might be due to greater weight loss in FTT babies, with babies requiring more time to catch up growth.

Most of our subjects gained weight after frenotomy and supplementation via the feeding tube device. Only the 11 subjects with FTT did not gain adequate weight. These infants with FTT had excessive weight loss during early life due to poor milk transfer from tongue- and lip-tie, and frenotomy and supplementation with feeding tube devices failed to adequately compensate. We provided early complementary feeding in these subjects. This approach was successful, and supplementation with feeding tube devices could later be stopped after improvements with breastfeeding and complementary feeding.

\section{Study Limitations}

The relatively small number of subjects, a lack of control group, and the descriptive, retrospective design limited this study. However, to further illustrate the validity of our approach, we present three example cases in Figure 3A-C. Figure 3A shows a case of baby girl who presented at 3 weeks with SWG. After frenotomy and supplementation, her nutritional status improved with good weight gain and supplementation was stopped by 2.5 months. She continued breastfeeding and grew well.

Figure $3 \mathrm{~B}$ shows a case of baby boy presenting at 3 weeks with FTT. He underwent frenotomy, and supplementation was started. Supplementation was stopped at 3 months, and he continued to grow well with only breastfeeding.

Figure 3C shows a case of baby boy presenting at 3 weeks with FTT. He underwent frenotomy, and supplementation was started. Her mother was working and could not take additional leave. Since he was still malnourished at 4 months of age, early complementary feeding was recommended. Supplementation was stopped at 4 months, and he continued to grow well with only breastfeeding and complementary feeding.

\section{Conclusions}

Tongue- and lip-tie can cause ineffective latching, poor milk transfer, and consequent SWG or FTT. The holistic management of tongue-tied babies with SWG or FTT consisting of frenotomy, supplementation, domperidone, and acupuncture can successfully improve nutritional status and simultaneously the mother's milk supply. We show that the baby's age will influence the outcome of treatment, with a younger age of starting treatment resulting in greater gains in weight. Babies with greater weight loss require patience and commitment for longer periods of treatment to increase weight. In a cohort of mothers and infants undergoing this regimen, babies were able to breastfeed without supplementation and gained weight.

\section{References}

Alamy, M., \& Bengelloun, W. A. (2012). Malnutrition and brain development: An analysis of the effects of inadequate diet during different stages of life in rat. Neuroscience $\mathcal{E}$ Biobehavioral Reviews, 36(6), 1463-1480. http://dx.doi.org/10.1016/j. neubiorev.2012.03.009

Albright, L. M. (2004). Domperidone in lactation: Use as a galactagogue. International Journal of Pharmaceutical Compounding, $8(5), 329-335$. 
Coryllos, E., Watson Genna, C., \& Salloum, A. (2004). Congenital tongue tie and its impact on breast feeding. Newsletter of the American Academy of Pediatrics, Summer.

Garbin, C. P., Sakalidis, V. S., Chadwick, L. M., Whan, E., Hartmann, P. E., \& Geddes, D. T. (2013). Evidence of improved milk intake after frenotomy: A case report. Pediatrics, 132(5), e1413-e1417. http://dx.doi.org/10.1542/peds.2012-2651

Gartner, L. M., Morton, J., Lawrence, R. A., Naylor, A. J., O'Hare, D., Schanler, R. J., Eidelman, A. I., \& American Academy of Pediatrics Section on Breastfeeding. (2005). Breastfeeding and the use of human milk. Pediatrics, 115(2), 496-506. http://dx. doi.org/10.1542/peds.2004-2491

Genna, C. W. (2013). Supporting sucking skills (2nd ed). Sudbury, MA: Jones and Bartlett.

Briggs, G. G., Freeman, R. K., \& Yaffe, S. J. (2011). Domperidone. In Drugs in pregnancy and lactation: A reference guide To fetal and neonatal Risk (9th ed.). Philadelphia, PA: Lippincott.

Horta, B. L., \& Victora, C. G. (2013). Long-term health effects of breastfeeding. World Health Organization, 129(8-9), 57-64.

Ivanovic, D. M., Leiva, B. P., Perez, H. T., Inzunza, N. B., Almagià, A. F., Toro, T. D., . . Bosch, E. O. (2000). Long-term effects of severe undernutrition during the first year of life on brain development and learning in Chilean high-school graduates. Nutrition, 16(11-12), 1056-1063. http://dx.doi.org/10.1016/ S0899-9007(00)00431-7

Jones, W., \& Breward, S. (2011). Use of domperidone to enhance lactation: what is the evidence? Community Practitioner: The Journal of the Community Practitioners' $\mathcal{E}$ Health Visitors' Association, 84(6), 35-37.

Kotlow, L. (2011). Infant reflux and aerophagia associated with the the maxillary lip-tie (Maxillary Frenum). Clinical Lactation, 2-4, 25-29.

Kumar, M., \& Kalke, E. (2012). Tongue-tie, breastfeeding difficulties and the role of frenotomy. Acta Paediatrica, 101(7), 687-689. http://dx.doi.org/10.1111/j.1651-2227.2012.02661.x

Mannel, R., \& Martens, P. J. M. W. (2013). Slow weight gain and failure to thrive. In Core curriculum for lactation consultant practice (3rd ed., p. 849). Burlington, MA: Jones and Bartlett.

Neifert, M. R. (2004). Breastmilk transfer: Positioning, latch-on, and screening for problems in milk transfer. Clinical Obstetrics and Gynecology, 47(3), 656-675. http://dx.doi.org/10.1097/01.grf. $\underline{0000136183.12304 .96}$

Powers, N. G. (2004). Low intake in the breastfeeding infant: Maternal and infant considerations. In Breastfeeding and human lactation (4th ed., pp. 325-363). Sudbury, MA: Jones and Bartlett.

Praborini, A., Purnamasari, H., Munandar, A., \& Wulandari, R. A. (2015). Early frenotomy improves breastfeeding outcomes for tongue-tied infants. Clinical Lactation, 6(1), 9-15. http://dx.doi. org/10.1891/2158-0782.6.1.9

Pransky, S. M., Lago, D., \& Hong, P. (2015). Breastfeeding difficulties and oral cavity anomalies: The influence of posterior ankyloglossia and upper-lip ties. International Journal of Pediatric Otorhinolaryngology, 79(10), 1714-1717. http://dx.doi.org/10. 1016/j.ijporl.2015.07.033

Riordan, J. (2005). Low intake in the breastfed infant: Maternal and infant considerations. In Breastfeeding and human lactation (pp. 277-307). Sudbury, MA: Jones and Bartlett.

Scrimshaw, N. S. (1998). Malnutrition, brain development, learning, and behavior. Nutrition Research, 18(2), 351-379. http://dx.doi.org/10.1016/S0271-5317(98)00027-X

The Academy of Breastfeeding Medicine Protocol Committee. (2009). ABM clinical protocol \#3: Hospital guidelines for the use of supplementary feedings in the healthy term breastfed neonate, Revised 2009. Breastfeeding Medicine, 4(3).

UNICEF. (2016). The community infant and young child feeding counselling package. Retrieved from https://www.unicef.org/ nutrition/index 58362.html

van Paassen, N., van der Starre, A. M., Hanff, L. M., Yap, S. C., Roorda Wierenga, T., \& Vermeulen, M. J. (2016). Domperidone to promote lactation. Nederlands tijdschrift voor geneeskunde, 160, D305.

Walker, M. (2011a). Beyond the initial 48-72 hours: Infant challenges. In Breastfeeding management for the clinician: Using the evidence (2nd ed., pp. 347-427). Sudbury, MA: Jones and Bartlett.

Walker, M. (2011b). Physical, medical, emotional and environmental challenges to the breastfeeding mother. In Breastfeeding management for the clinician: Using the evidence (pp. 581-637). Sudbury, MA: Jones and Bartlett.

Wambach, K. J. R. (2014). Low intake in the breastfed infant:maternal and infant considerations. In Breastfeeding and Human Lactation (5th ed., pp. 359-401). Sudbury, MA: Jones and Bartlett.

Wen, L. M., Baur, L. A., Simpson, J. M., Rissel, C., \& Flood, V. M. (2011). Effectiveness of an early intervention on infant feeding practices and "tummy time": A randomized controlled trial. Archives of pediatrics $\mathcal{E}$ adolescent medicine, 165(8), 701-707. http://dx.doi.org/10.1001/archpediatrics.2011.115

World Health Organization. (2000). Complementary feeding family foods for breastfed children. Retrieved from http://www.who. int/nutrition/publications/infantfeeding/WHO NHD 00.1/ en/

World Health Organization. (2016). Infant and young child feeding. Retrieved from http://www.wpro.who.int/nutrition wpr/ publications/infantchildfeeding.pdf

Yelland, S. (2005). Insufficient lactation. In Acupunture in midwifery (2nd ed., p. 64). London, UK: Elsevier.

Acknowledgments. The authors gratefully acknowledge the CEO and staff of Kemang Medical Care Mother and Child Hospital, Jakarta, Indonesia; Mrs Titi Mulyani, S.Si, Apt., MARS, from Islamic University Syarif Hidayatullah, Jakarta, Indonesia; and the Praborini Lactation Team, Jakarta and West Java, Indonesia. 


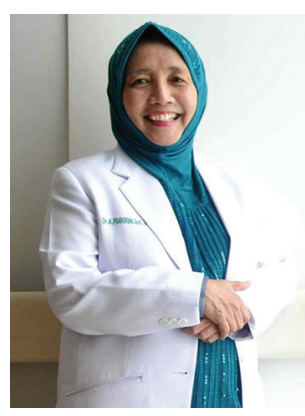

Asti Praborini, MD, IBCLC, RLC, is a granny of a successful breastfeeding mother. Having 28 years of experience as pediatrician convinced her that nothing is more important and valuable than breastfeeding for both mother and baby. She built the first dreamed hospital-based lactation team in Indonesia that works ultimately to help mother breastfeed her baby. She is practicing frenotomy for anterior as well as posterior tongue-tie and lip-tie.

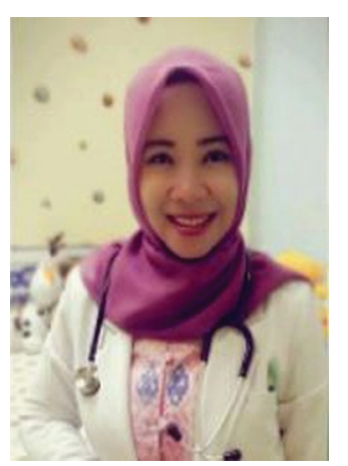

Anjar Setiani, MD, is a pediatrician in KMC Women and Children Hospital, Jakarta, Indonesia. She contributes in establishing the hospital-based lactation team in Indonesia. She is practicing frenotomy for tongue-tie and lip-tie and now helping many mothers to achieve their breastfeeding goals.

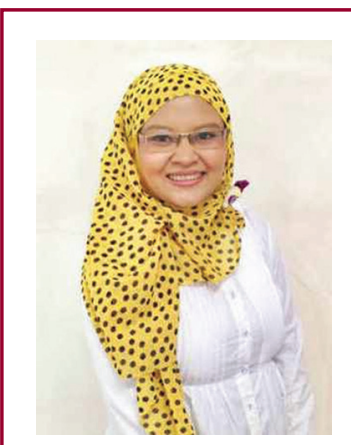

Agusnawati Munandar, MD, IBCLC, RLC, CIMI, is a successful breastfeeding mother of two. She is working in a lactation clinic of Baby-Friendly hospitals and earned her IBCLC degree in 2013.

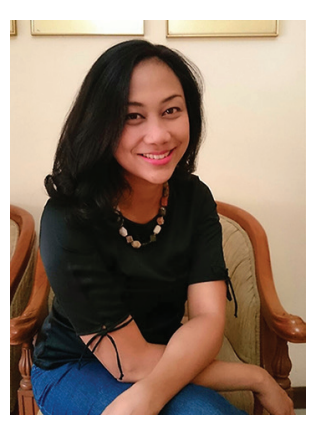

Ratih Ayu Wulandari, MD, IBCLC, RLC, entered the lactation field once she realized that breastfeeding mothers need help and support. Having breastfed her tongue-tie baby she understands the pain, and supports early frenotomy. She is now working with the lactation team and practicing frenotomy for anterior and posterior tongue-tie and lip-tie. She believes that attachment parenting is the best way to nurture a child and shares her thoughts on her blog posting www. menjadiibu.com. 